\title{
Facilidades/dificuldades vivenciadas por graduandos de uma universidade pública na elaboração da evolução de enfermagem
}

\section{Public university students' ease and/or difficulties in preparing nursing progress notes}

\author{
Facilidades/dificultades experimentadas por los estudiantes de una universidad pública en la \\ elaboración de la evolución de enfermería
}

\author{
Daniela Maack Silveira'; Mariângela Magalhães"II; Maria Cristina Chagas ${ }^{\text {III }}$; \\ Silomar Ilha ${ }^{I V}$; Glaucia Dal Omo Nicolav ; Fabiani Weiss Pereira ${ }^{\mathrm{Vl}}$
}

\begin{abstract}
RESUMO: Objetivou-se conhecer os fatores que facilitam/dificultam a elaboração da evolução de enfermagem por graduandos de uma universidade pública. Pesquisa descritiva, de abordagem qualitativa, realizada no mês de abril de 2012, por meio de questionário semiestruturado com 13 graduandos do oitavo e nono semestres do Curso de Graduação em Enfermagem de uma Universidade Federal do Estado do Rio Grande do Sul. Para análise dos dados, utilizou-se a análise de conteúdo temática. Como resultados, emergiram três categorias - Evolução de enfermagem: instrumento de comunicação e cuidado; Organização e valorização do tempo; e Ensino do processo de enfermagem na graduação. Conclui-se que houve divergências no ensino em relação aos registros e que a falta de tempo e a ausência de registros da equipe de enfermagem dos campos de prática representam dificuldades na efetivação da evolução de enfermagem.
\end{abstract}

Palavras-Chave: Processo de enfermagem; estudantes de enfermagem; registros de enfermagem; evolução do cliente.

\begin{abstract}
This qualitative, descriptive study to discover the factors that facilitate/hinder preparation of nursing progress notes by nursing undergraduates at a public university was conducted in April 2012 by means of a semi-structured questionnaire applied to $138^{\text {th }}$ and $9^{\text {th }}$ semester undergraduate nursing students at Rio Grande do Sul Federal University. From data analysis using thematic content analysis, three categories emerged: nursing progress notes, an instrument for communication and care; organization and optimization of time; and the undergraduate nursing teaching process. It was concluded that the teaching displayed divergences on record keeping, while lack of time and the absence of nursing staff record-keeping during practical activities hindered effective preparation of nursing progress notes.
\end{abstract}

Keywords: Nursing process, nursing students, nursing records; client progress.

RESUMEN: El estudio tuvo como objetivo conocer los factores que facilitan/dificultan la elaboración de la evolución de la enfermería a través de los estudiantes de una universidad pública. Investigación descriptiva con enfoque cualitativo, realizada en abril de 2012, por medio de cuestionario semiestructurado junto a 13 estudiantes del octavo y noveno semestre de la graduación en enfermería de una Universidad Federal de Rio Grande do Sul. Para analizar los datos, se ha utilizado el análisis de contenido temático. De los resultados, surgieron tres categorías: Evolución de Enfermería - instrumento de comunicación y atención; Organización y valoración del tiempo y; Enseñanza del proceso de enfermería en el curso de graduación. Se concluye que hubo divergencias en la enseñanza en relación con los registros y que la falta de tiempo y la ausencia de registros del equipo de enfermería de los campos de entrenamiento representan dificultades para hacer efectiva la evolución de la enfermería.

Palabras Clave: Proceso de enfermería, estudiantes de enfermería, registros de enfermería; evolución del cliente.

\section{INTRODUÇÃO}

O processo de enfermagem (PE) destaca-se como uma tecnologia do cuidado o qual orienta a sequência do raciocínio lógico, melhorando a qualidade do cuidado por meio da sistematização da avaliação clínica, servindo, também, de fundamentação para a educação, pesquisa e gerenciamento. Deve ser utilizado pelos enfermeiros, pois, por meio dele, há a possibilidade de organizar e garantir a continuidade das informações da equipe de enfermagem,

'Enfermeira. Programa de Pós-Gradução em Enfermagem, Universidade Federal do Rio Grande. Brasil. E-mail: dmaacksilveira@yahoo.com.br. IIEnfermeira. Mestre em Enfermagem. Docente do Curso de Enfermagem da Universidade Federal do Rio Grande. Brasil. E-mail: mariprof43@yahoo.com.br. IIIEnfermeira. Mestranda, Programa de Pós-Gradução em Enfermagem, Universidade Federal do Rio Grande. Brasil. E-mail: maria25cris@yahoo.com.br.

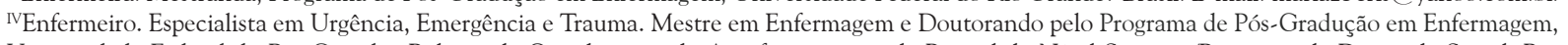
Universidade Federal do Rio Grande. Bolsista da Coordenação de Aperfeiçoamento de Pessoal do Nível Superior/Programa de Demanda Social. Rio Grande do Sul, Brasil. E-mail: silo_sm@hotmail.com.

vEnfermeira. Mestre em Enfermagem pelo Programa de Pós-Gradução em Enfermagem, Universidade Federal do Rio Grande. Brasil. E-mail: glaucianicola@hotmail.com.

vIEnfermeira. Mestranda do Programa de Pós-Gradução em Enfermagem, Universidade Federal do Rio Grande. Bolsista da Coordenação de Aperfeiçoamento de Pessoal do Nível Superior/Programa de Demanda Social. Rio Grande do Sul, Brasil. E-mail: enffabiweiss@hotmail.com. 
permitindo avaliar a sua eficácia e efetividade ${ }^{1}$. Além disso, o PE auxilia nas inter-relações entre os sujeitos envolvidos no cuidar-cuidado, configurando-se numa alternativa para reaproximar o enfermeiro de sua clientela 2 .

O PE foi introduzido no Brasil, na década de 70, por Wanda de Aguiar Horta, e é composto por seis fases: histórico de enfermagem, diagnóstico de enfermagem, plano assistencial, prescrição de enfermagem ou plano de cuidados, evolução de enfermagem e prognósticos de enfermagem ${ }^{3}$. Atualmente, o $\mathrm{PE}$ organiza-se em cinco etapas inter-relacionadas e interdependentes: histórico de enfermagem, diagnósticos de enfermagem, planejamento de enfermagem, implementação e avaliação de enfermagem ${ }^{4}$.

Dessa forma, faz-se pertinente explicitar a finalidade dos registros de enfermagem, pois esses são considerados o referencial do processo de trabalho de enfermagem. Por meio dele é possível assegurar a comunicação entre os membros da equipe de saúde e, assim, garantir a continuidade das informações para as diversas áreas, favorecendo o ensino, a pesquisa, a verificação de aspectos legais e a garantia de assistência ao cliente ${ }^{5}$. Além disso, sabe-se da importância de todas as etapas do PE, principalmente da Evolução de Enfermagem (EE), que é o relato diário ou periódico das mudanças sucessivas que ocorrem no ser humano, enquanto estiver sob assistência profissional ${ }^{3: 67}$.

O ensino do PE, na Universidade Federal, onde foi desenvolvida esta pesquisa, faz parte da grade curricular, desde a criação do curso. O referencial teórico utilizado é o modelo de Wanda Horta, o qual se baseia na Teoria das Necessidades Humanas Básicas (TNHB). O PE, inicialmente, era ministrado na disciplina de Fundamentos de Enfermagem, nos terceiro e quarto semestres; posteriormente, passou a ser ministrado na disciplina de Introdução à Enfermagem. A disciplina possui carga horária total de 45 horas, ministradas em 3 horas semanais. Destas, aproximadamente 15 horas são de atividades práticas. $\mathrm{O}$ atual Projeto Político-Pedagógico do curso de enfermagem da instituição do estudo tem o PE como um dos eixos condutores do currículo, sendo a sua padronização nas disciplinas uma grande preocupação da Coordenação do Curso e Núcleo Docente Estruturante ${ }^{6}$.

A evolução de enfermagem sugerida pela Escola de Enfermagem, onde foi desenvolvida esta pesquisa, baseia-se no modelo SOAP - Subjetivo: Informações fornecidas pelo paciente ou familiares como a queixa principal e outras informações. Objetivo: Dados factuais e mensuráveis que se obtêm durante o exame, como os sinais e sintomas observados, sinais vitais e valores de exames laboratoriais. Avaliação: As conclusões baseadas nos dados subjetivos e objetivos coletados e formuladas como diagnósticos de enfermagem. Plano: Estratégia para aliviar o problema do paciente. Este plano deve incluir ações de curto e longo prazos ${ }^{6}$.
Espera-se contribuir para reorientação do ensino da enfermagem e construção de novos conhecimentos sobre a temática, além de beneficiar, indiretamente, os clientes, fornecendo-lhes uma melhor qualidade da assistência. Esta é a relevância deste estudo.

Dessa forma, questiona-se: Quais os fatores que facilitam/dificultam a elaboração da evolução de enfermagem pelos graduandos? Na tentativa de responder a esse questionamento, objetivou-se, com este estudo, identificar os fatores que facilitam/dificultam a elaboração da evolução de enfermagem pelos graduandos de uma universidade pública.

\section{REVISÃO DE LITERATURA}

$O$ processo de enfermagem é a base científica que sustenta as ações de enfermagem, sendo considerado uma forma ordenada e sistemática do agir do enfermeiro para identificar e resolver problemas levantados junto aos pacientes. A sua implementação tem como vantagens: facilitar a documentação de dados, subsidiar o levantamento de problemas e elaboração das intervenções de enfermagem de forma sistemática, elaborar uma linguagem comum de enfermagem que facilite a comunicação com a equipe e desenvolver meios de avaliação da assistência prestada? .

\section{As etapas do processo de enfermagem são:}

Histórico de enfermagem - É uma fase imprescindível para que ocorra o sucesso das demais fases. Os dados são coletados por meio da entrevista, observação e exame físico. Assim, o histórico de enfermagem resulta de informações objetivas e subjetivas relatadas pelo paciente, na entrevista. Deve ser conciso, sem repetições, permitindo programar cuidados imediatos ${ }^{8}$.

Diagnóstico de enfermagem - Consiste na interpretação e agrupamento dos dados coletados na primeira etapa e que culmina com a tomada de decisão sobre os conceitos diagnósticos que guiarão o planejamento e a implementação da assistência. Representa, com mais exatidão, as respostas do indivíduo ou coletividade humanas, em um dado momento do processo saúde-doença, e se constitui a base para as futuras ações (intervenções) junto ao cliente, visando superar os problemas identificados ${ }^{4}$.

Planejamento de enfermagem - Consiste na determinação global dos resultados esperados pelo paciente e das intervenções específicas de enfermagem determinadas pelas necessidades humanas, identificadas na etapa diagnóstica. Essa fase é realizada mediante um roteiro diário de enfermagem que coordena as ações da equipe. O planejamento envolve ações de encaminhamento, supervisão, orientação, ajuda e execução de intervenções/ cuidados de enfermagem, constituindo-se no somatório de todas as ações desenvolvidas pela equipe de enfermagem no atendimento ao paciente ${ }^{3}$. 
Implementação de enfermagem - consiste na realização das ações ou intervenções determinadas na etapa de planejamento de enfermagem ${ }^{4}$. Durante essa fase, o enfermeiro continua coletando dados e validando o planejamento realizado, uma vez que é imprescindível a atualização das informações. A prescrição de enfermagem é o roteiro diário que coordena a ação da equipe de enfermagem nos cuidados adequados ao atendimento das necessidades básicas e específicas do ser humano. A prescrição de enfermagem deverá ter como base os cuidados prioritários ${ }^{3}$.

Avaliação de enfermagem - É um processo deliberado, sistemático e contínuo de verificação de mudanças nas respostas da pessoa, família ou coletividade humana, em um dado momento do processo saúde-doença, para determinar se as ações ou intervenções de enfermagem alcançaram o resultado esperado ${ }^{4}$. Após a implementação do cuidado, cada etapa deve ser avaliada, a fim de verificar se os diagnósticos ainda persistem, se as metas foram atingidas ou se estão de acordo com a realidade do paciente e se ele está aceitando as intervenções que estão sendo executadas ${ }^{3}$.

\section{Metodologia}

Trata-se de uma pesquisa descritiva, de abordagem qualitativa ${ }^{9}$, realizada com 13 graduandos de enfermagem do oitavo e nono semestres do Curso de Graduação em Enfermagem de uma Universidade Federal do Estado do Rio Grande do Sul, sendo oito graduandos do oitavo e cinco do nono semestre.

Os critérios de inclusão dos sujeitos do estudo foram: estar cursando o oitavo ou nono semestre do Curso de Graduação em Enfermagem, estar realizando Estágio Supervisionado I e II nas unidades de clínica médica, clínica cirúrgica, pediatria, maternidade ou tratamento intensivo, no mês de abril de 2012.

Optou-se por realizar a pesquisa com graduandos de oitavo e nono semestres em razão destes estarem em processo de conclusão de curso e com mais experiência em relação à aplicação do $\mathrm{PE}$, em especial os registros da evolução de enfermagem. Dessa forma, foram excluídos da pesquisa os graduandos de enfermagem do primeiro ao sétimo semestre e aqueles que, por alguma razão, não estivessem realizando Estágio Supervisionado I e II.

A coleta dos dados ocorreu no mês de abril de 2012, por meio de questionário semiestruturado. No primeiro momento, foi entregue aos sujeitos questionário estruturado com questões abertas sobre o PE, sendo agendado com o graduando um horário para seu recolhimento. No segundo momento, o questionário respondido foi lido juntamente com o respondente, para sua validação, bem como para esclarecer dúvidas e aprofundar as respostas.

Os dados foram analisados por análise de conteúdo de Bardin ${ }^{10}$, seguindo as três etapas do método. Primeira- mente, buscou-se fazer uma leitura exaustiva dos dados, seguida da organização do material e a formulação de hipóteses. Na sequência, foi realizada a exploração do material, ou seja, buscou-se codificar os dados brutos. $\mathrm{Na}$ terceira e última fase, os dados foram interpretados e delimitados os eixos temáticos pela compreensão dos significados emergentes dos depoimentos.

Foram considerados os preceitos éticos e legais que envolvem a pesquisa com seres humanos, conforme a Resolução n ${ }^{\circ}$ 466/2012 do Ministério da Saúde ${ }^{11}$. Foi distribuído, anteriormente, o Termo de Consentimento Livre e Esclarecido para os participantes da pesquisa, sendo este em duas vias, ficando uma em poder do participante e outra do pesquisador. Manteve-se o anonimato dos depoentes, que foram identificados por nomes de estrelas. $\mathrm{O}$ projeto de pesquisa foi aprovado pelo Comitê de Ética e Pesquisa na Área da Saúde da Universidade Federal do Rio Grande (CEPAS/FURG), sob o número 188/2011.

\section{Resultados}

A análise dos dados gerou três categorias - Evolução de enfermagem: instrumento de comunicação e cuidado; Organização e valorização do tempo; e Ensino do PE na graduação.

\section{Evolução de enfermagem: instrumento de co- municação e cuidado}

Conforme as respostas dos questionários, os graduandos consideram importante o registro da Evolução de Enfermagem no prontuário do cliente, sendo enfatizado como um instrumento de comunicação entre os profissionais de saúde e da própria equipe de enfermagem. É onde o profissional registra todas as informações relativas ao cuidado, tornando visível seu trabalho e, sobretudo, oportunizando a avaliação do cliente e a definição das condutas escolhidas, conforme as seguintes falas:

\section{O registro da evolução de enfermagem é importante para que o enfermeiro do plantão seguinte possa ficar informado do que ocorreu durante o turno. (Vega) \\ Facilita a comunicação [entre os profissionais de saúde] dos diferentes turnos [de trabalho]. (Spica) \\ É através da evolução de enfermagem que podemos registrar todos os cuidados realizados [pela enfermagem], mostra o trabalho efetivo. (Alhena)}

Apesar de saberem que a evolução de enfermagem é um importante instrumento de comunicação e cuidado, alguns graduandos referem que a equipe de saúde das unidades não valoriza o PE e pouco se utiliza dos registros de enfermagem realizados pelos acadêmicos, chegando a oferecer resistência a sua realização.

[...] muitas vezes a evolução de enfermagem não vai ser lida pelos colegas de profissão. (Sírius) 
Resistência dos profissionais em realizar as evoluções [...] A evolução de enfermagem está sempre em segundo plano. (Nunki)

Os registros de enfermagem, quando não realizados no prontuário do cliente, além de não constituírem um documento legal, impedem a efetiva comunicação entre a equipe, como se observa no local de estudo que ainda utiliza o tradicional livro de ocorrências.

Já na construção do registro escrito, não tem facilidade para sentar e evoluir um paciente de forma adequada com o processo de enfermagem completo, pela demanda da unidade, então acaba que registramos apenas as alterações diárias mais relevantes em um caderno preto e as evoluções não são realizadas. (Capella)

\section{Organização e valorização do tempo}

Os graduandos de enfermagem percebem o PE como um instrumento de comunicação e de cuidado. No entanto, oito dos 13 sujeitos do estudo referiram dificuldades em relação à organização do tempo. Alguns mencionaram não ter tempo para evoluir e outros acreditam que perdem muito tempo com a evolução, mencionando, ainda, que a equipe da unidade desvaloriza o tempo que eles utilizam para realizar os registros, chegando a deixá-los desconfortáveis no momento de executá-los.

Quando elaboro minhas evoluções de enfermagem não são completas por falta de tempo. Sobrecarregamo-nos de tarefas e falta tempo para fazer uma boa evolução. (Vega)

Uma dificuldade é a elaboração da evolução de enfermagem em tempo longo; gostaria de elaborar em tempo mais curto. (Altair)

Facilitam [a equipe de enfermagem] muito pouco, pois ainda não se tornou uma prática para todos. Assim, a diferença de quem faz causa desconforto para os outros. (Spica)

Há, também, a ausência de prioridade, por parte dos graduandos participantes deste estudo, conforme observa-se na seguinte fala:

A dificuldade é a demanda da unidade e, ao mesmo tempo, a gana de aprender em pouco espaço de tempo o que ainda não sentimos segurança como gostaríamos; dessa forma, acaba se dando prioridades para outras atividades. (Capella)

\section{Ensino do PE na graduação}

Quando questionados acerca do ensino do PE, todos os graduandos relataram que tal processo facilitou a elaboração da evolução de enfermagem, dando subsídios para construção dos registros; por outro lado, muitos deles citaram que existem divergências, por parte dos professores, em relação à realização dos registros, o que dificulta sua execução.

Sinto-me segura em elaborar registros de enfermagem após o ensino e abordagem ministrados na academia. (Nunki)
Cada disciplina que passamos, bem como cada profissional [professor], ensinou sua forma de realizar o registro e isso acaba criando uma confusão na elaboração [ $\mathrm{da}$ evolução]. (Nunki)

Apenas na questão de cada professor exigir de uma forma, isso acaba dificultando o aprendizado. (Polaris)

A percepção dos graduandos deste estudo reitera esta questão, visto que consideram as diferentes formas de abordagem como uma dificuldade. Entretanto, alguns destacam que a compreensão do PE acontece ao longo do curso, como observado na seguinte fala:

$\mathrm{Na}$ disciplina de SAE, não ficou claro o diagnóstico de enfermagem. Fui entender no sexto semestre, na disciplina de gerontogeriatria. (Adhara)

Apesar de considerarem que o ensino do PE, na instituição em estudo, facilitou a elaboração da evolução de enfermagem, certos acadêmicos destacaram sua dificuldade em registrá-la, especialmente os dados subjetivos.

Acredito que a parte objetiva da evolução seria uma facilidade. Já a dificuldade seria pela parte subjetiva, pois temos que atentar para o comportamento do paciente comparando com a veracidade de suas respostas. (Alhena)

Minhas facilidades estão relacionadas aos cuidados que eu realizo, tudo o que eu observo torna-se mais fácil de registrar do que aquilo que o paciente relata. Tenho dificuldades de elaborar e registrar a evolução quando só tenho informações ditas pelo paciente. (Adhara)

Nos depoimentos, não fica claro se os aspectos subjetivos são mais difíceis de registrar por não terem sido compreendidos suficientemente pelo graduando (dificuldade na comunicação) ou por serem mais difíceis de avaliar (dificuldade no raciocínio clínico).

\section{Discussão}

Os registros efetuados pela equipe de enfermagem têm finalidade de fornecer informações sobre assistência prestada ao cliente, assegurando a comunicação entre os membros da equipe de saúde e garantindo a continuidade e atualização das informações. Oferecem respaldo legal e, consequentemente, segurança, pois constituem o único documento que relata todas as ações da enfermagem junto ao cliente ${ }^{12}$. Estudos evidenciam o ambiente hospitalar como um local de constante troca de informações e experiências por meio de inter-relações, sendo a comunicação uma ferramenta ideal para a efetividade dos serviços ${ }^{2,5}$.

Nesse sentido, a anotação de enfermagem é de extrema importância, pois sua ausência pode expor os clientes a riscos, não garantindo, assim, a qualidade e assistência adequadas ${ }^{5}$. Pesquisa desenvolvida no Reino Unido com 19 professores de uma escola de enfermagem destaca a importância da pesquisa com estudantes e professores acerca do PE e da realização adequada das anotações de enfermagem, pois, por meio dos registros, 
é possível o desenvolvimento da sistematização da assistência de enfermagem ${ }^{13}$.

A inadequação e a insuficiência dos registros podem comprometer a assistência ao cliente, pois inviabilizam que as informações corretas sejam transmitidas com segurança aos demais profissionais, além de manterem invisível o fazer da enfermagem perante a lei e demais profissionais da equipe de saúde ${ }^{14}$. Um estudo realizado em uma UTI geral salienta que informações incorretas ou mal registradas podem intervir negativamente na recuperação do cliente, e remeter à hipótese de que o cuidado não foi contemplado de maneira global e individualizada 5 .

Os sujeitos deste estudo referiram dificuldades para efetuar o PE em decorrência da falta de tempo /muito tempo dispensado ao fazê-lo, dificultando o fazer de outras atividades. Um estudo realizado no Reino Unido, com 19 professores, evidenciou que, na prática, apesar de os docentes enfatizarem e elaborarem estratégias durante a formação acadêmica para o ensino do PE, a maioria dos enfermeiros não as utiliza no seu exercício profissional ${ }^{14}$.

Em contraste a esse estudo, uma pesquisa realizada na Bolívia evidenciou que o PE está relacionado à motivação de trabalhadores e da chefia da instituição, além da universidade local, fazendo com que as estratégias sugeridas contemplem ações mais diretas para a operacionalização do PE, com o desenvolvimento de instrumentos, formulários de preenchimento para a avaliação inicial de enfermagem e a alteração dos planos de ensino do Curso de Graduação em Enfermagem ${ }^{15}$. No Brasil, existem diversos estudos apontando para certas dificuldades da prática assistencial, como o desenvolvimento incompleto do $\mathrm{PE}$, registros incompletos, entre outros ${ }^{5-16,17}$.

Essas dificuldades podem ser atribuídas, entre outros fatores, à sobrecarga de trabalho do enfermeiro, evidenciando a importância da reflexão acerca desta questão. O enfermeiro, por sobrecarga diária de trabalho, muitas vezes, acaba não ofertando a atenção devida ao cliente em relação às anotações de enfermagem, o que não se justifica, pois os registros servem para certificar que o cuidado foi realizado e, também, como documento legal para segurança do cliente e do próprio profissional que anota $^{5}$. Além disso, pode-se relacionar a falta de tempo dos enfermeiros para efetuar os registros de enfermagem como consequência da ausência de prioridade desta tarefa no seu fazer. Assim, eles devem buscar alternativas que viabilizem uma prática qualificada que também está pautada pela qualidade dos registros efetuados ${ }^{14}$.

Os sujeitos deste estudo referiram que existem divergências por parte dos professores em relação à realização dos registros, o que dificulta tal execução. Em uma pesquisa realizada com docentes da mesma instituição em estudo, pode-se perceber que não existe uniformidade na linguagem, em relação ao processo de enfermagem e suas etapas, inclusive na abordagem do tema, o que acarreta dificuldades tanto no ensino quanto no aprendizado ${ }^{18}$.
Pesquisa realizada em três instituições de Ensino Superior, no município de João Pessoa/PB, evidenciou que, apesar da preocupação no resgate do binômio teoria e prática, a observação livre permitiu inferir que há dissociação entre o que se aprende na teoria e o que se observa na prática. Dessa forma, os docentes referem certo grau de dificuldade quanto ao ensino, principalmente do diagnóstico de enfermagem, devido às competências necessárias a sua execução. Ressalta, ainda, que há uma fragmentação na construção do conhecimento e desarticulação no ensino do PE, acarretando dificuldades de percepção dos discentes na integração entre as disciplinas do curso no que diz respeito ao ensino desse processo ${ }^{2}$.

Nessa perspectiva, a valorização da subjetividade depende de estímulos prévios, como a análise de fenômenos por diferentes olhares, localizando-o no tempo e no contexto social, buscando os mais diversos fatores relacionais, bem como as possíveis repercussões futuras ${ }^{19}$. Com o pensamento crítico, o indivíduo revela condições de exercitar outras operações mentais tais como a análise, síntese, avaliação, interpretação, aplicação e tomada de decisão, indispensáveis na aplicação do PE? .

Os registros de enfermagem completos são considerados um dos principais indicadores de qualidade da assistência de enfermagem. A Decisão n ${ }^{\circ} 115 / 2006$, do Conselho Regional de Enfermagem do Rio Grande do Sul, normatiza que os registros de enfermagem devem ser legíveis, completos, claros, concisos, objetivos, pontuais e precedidos de data e hora ${ }^{12}$. O PE é um processo sistemático e contínuo de verificação de mudanças nas respostas da pessoa, família ou coletividade humana, em um dado momento do processo saúde-doença, para determinar se as ações ou intervenções de enfermagem alcançaram o resultado esperado ${ }^{4}$.

\section{Conclusão}

Considera-se satisfatória a realização deste estudo, pois foi possível identificar que os sujeitos valorizam o PEe reconhecem a importância dos registros para enfermagem e para o paciente. Reforçam que o PE proporciona organização do trabalho, continuidade ao cuidado prestado, de forma a garantir assistência mais qualificada, permitindo a visualização do trabalho da enfermagem e, com isso, aumento do reconhecimento profissional.

Os sujeitos destacaram dificuldades para elaborar a evolução de enfermagem em suas atividades práticas, em função do tempo, ou seja, relataram que a demanda de serviços da unidade dificulta acompanhar a evolução dos pacientes de forma completa. Além disso, mencionaram desconforto perante as equipes de enfermagem dos campos de prática que não utilizam os registros do $\mathrm{PE}$. Os sujeitos apontaram o ensino do PE como facilitador na elaboração dos registros de enfermagem e que deve ser implementado desde as séries iniciais, porém relataram 
que há divergências por parte dos professores em relação aos registros, o que dificulta a sua execução.

Como limitação deste estudo, aponta-se o tempo para realização da pesquisa, que precisou respeitar o prazo de conclusão, e, dessa forma, nem todos os dados obtidos chegaram a ser categorizados. Os dados não trabalhados suficientemente deixam brecha para a realização de outros estudos com aprofundamento da mesma temática. A integração desta pesquisa com outros trabalhos realizados com docentes, acadêmicos e enfermeiras dão subsídios para uma questão de pesquisa que vem sendo amplamente estudada na instituição.

Espera-se que este trabalho traga contribuições para o ensino do PE e que os acadêmicos, em especial os estagiários de final de curso, passem a assumir mais o seu papel na aplicação desse processo, despertando o interesse e a compreensão da equipe de enfermagem dos campos de prática por essa tecnologia.

\section{REFERÊNCIAS}

1.Dal Sasso GTM, Barra DCC, Paese F, Almeida SRW, Rios GC, Marinho MM, Debétio MG. Processo de enfermagem informatizado: metodologia para associação da avaliação clínica, diagnósticos, intervenções e resultados. Rev esc enferm USP [Internet]. 2013 [citado em 18 jul 2015]; 47 (1):242-9. Disponível em: http://www.scielo. br/pdf/reeusp/v47n1/a31v47n1.pdf

2.Leadebal ODCP, Fontes WD, Silva CC. Ensino do processo de enfermagem: planejamento e inserção em matrizes curriculares. Rev esc enferm USP [Internet]. 2010 [citado em 25 nov 2014]; 44:190-8. Disponível em: http://www.scielo.br/scielo.php?script=sci_arttext \&pid $=$ S0080-62342010000100027

3.Horta WA, Castellanos BP. Processo de enfermagem. São Paulo: EPU; 1979.

4.Conselho Federal de Enfermagem. Resolução n 358/09, dispõe sobre o processo de enfermagem nas Instituições de Saúde Brasileiras. Rio de Janeiro: COFEn; 2009. [citado em 17 ago 2015]. Disponível em: http://site.portalcofen. gov.br/node/4384

5.Cordeiro FF, Dyniewicz AM, Kalinowski LC. Auditoria em registros de enfermagem em unidade de terapia intensiva. Rev enferm UFPE on line [Internet]. 2011 [citado em 14 jul 2015]; 5:1187-92. Disponível em: http://www.revista.ufpe.br/revistaenfermagem/index. $\mathrm{php} /$ revista/article/view/1552

6.Universidade Federal do Rio Grande. Projeto Político Pedagógico do Curso de Enfermagem. Rio Grande (RS): FURG; 2004. [citado em 15 set 2015]. Disponível em: http:// www.eenf.furg.br/images/stories/pppenf.doc.

7.Schulz RS, Silva MF. Análise de evolução dos registros de enfermagem numa unidade cirúrgica após implantação do método SOAP. Revista Hospital Universitário Pedro Ernesto/UERJ. [Internet]. 2011 [citado em 15 jun 2015]; 10(1):91-9. Disponível em: http://revista.hupe.uerj.br/ detalhe artigo.asp?id=127
8.Cossa RMV. O ensino do processo de enfermagem em uma universidade pública e hospital universitário do sul do Brasil na perspectiva de seus docentes e enfermeiros. [dissertação de mestrado] Porto Alegre (RS): Universidade Federal do Rio Grande do Sul; 2011.

9.Minayo MCS. O desafio do conhecimento: pesquisa qualitativa em saúde. $11^{\mathrm{a}}$ ed. São Paulo: Hucitec; 2008.

10.Bardin L. Análise de conteúdo. $4^{\mathrm{a}}$ ed., Lisboa (Pt): Edições 70; 2009.

11.Conselho Nacional de Saúde (Br). Resolução n ${ }^{\circ} 466$, de 12 de dezembro de 2012. Diretrizes e normas regulamentadoras de pesquisa em seres humanos. Brasília (DF): CNS; 2012. 12.Conselho Regional de Enfermagem (RS). Decisão COREN-RS n ${ }^{\circ}$ 115/2006. [internet] Porto Alegre (RS): COREn-RS; 2006 [citado em 20 jul 2015]. Disponível em: http://www.portalcoren-rs.gov.br/index.php?categoria = pro fissional\&pagina $=$ decisoes

13.Torrance C, Mansell I, Wilson C. Learning objects? Nurse educators' views on using patients for student learning: ethics and consent. Education for Health [Internet]. 2012 [citado em 14 jul 2013]; 25(2):92-7. Disponível em: http://www.educationforhealth.net/temp/EducHealth25292-3760981_102649.pdf

14.Pimpão F D, Lunardi Filho WD, Vaghetti HH, Lunardi VL. Percepção da equipe de enfermagem sobre seus registros: buscando a sistematização da assistência de enfermagem. Rev enferm UERJ. [Internet]. 2010 [cited in 2015 Jul 10]; 18:405-10. Disponível em: http://www.facenf.uerj.br/v18n3/ v18n3a12.pdf

15.Fernández-Sola C, Granero-Molina J, Aguilera-Manrique G, Peredo-de Gonzales MH, Castro-Sánchez AM, Pérez Galdeano A. Strategies to develop the nursing process and nursing care plans in the health system in Bolivia. International Nursing Review [Internet]. 2011 [cited in 2015 Jul 10]; 58:3:392-9. Available from: http://onlinelibrary.wiley. com/doi/10.1111/j.14667657.2011.00884.x/abstract;jsessio nid=0141477C542BEFAF6BA5DB48E58BA0EF.d04t03 16.Pokorski S, Moraes MA, Chiarelli R, Costanzi AP, Rabelo ER. Processo de Enfermagem: da literatura à prática. O quê de fato nós estamos fazendo. Rev Latino-am Enfermagem [Internet]. 2009 [citado em 10 jul 2015]; 17:302-7. Disponível em: http://www.scielo.br/scielo. php? pid $=$ S0104-11692009000300004\&script $=$ sci abstract\&tlng=pt.

17.Garcia TR, Nóbrega MML. Processo de enfermagem: da teoria à prática assistencial e de pesquisa. Esc Anna Nery [Internet]. 2009 [citado em 10 jul 2015];13:188-93. Disponível em: http://www.scielo.br/pdf/ean/v13n1/v13n1a26 18.ROSA CS. Ensino do processo de enfermagem: metodologias utilizadas pelos docentes [Trabalho de conclusão de curso] Rio Grande (RS): Universidade Federal do Rio Grande; 2012.

19.Aquino DR. Construção e implantação da prescrição de enfermagem informatizada em uma UTI. [dissertação de mestrado] [internet] Rio Grande (RS): Fundação Universidade Federal do Rio Grande; 2004. [citado em 10 mai 2015]. Disponível em: http://www.ppgenf.furg.br/ images/daiseaquino.pdf 